\title{
SiMULACIÓN NUMÉRICA DEL FLUJO SUBEXPANDIDO EN LA TOBERA CÓNICA EXPERIMENTAL HELIOS-X
}

\author{
NUMERICAL SIMULATION OF THE \\ UNDER-EXPANDED FLOW IN THE \\ EXPERIMENTAL CONICAL NOZZLE HELIOS-X
}

\author{
San Luis B. Tolentino Masgo ${ }^{1,2, *}$, Richard Nakka ${ }^{3}$, Simón Caraballo ${ }^{4}$, Jorge Mírez ${ }^{2,5}$
}

Recibido: 11-03-2020, Revisado: 11-03-2020, Aprobado tras revisión: 17-10-2020

\section{Resumen}

Estudios numéricos del campo de flujo para toberas convergentes-divergentes con longitud de garganta, han reportado fluctuaciones del flujo con ondas de choque oblicuo en la sección de la garganta, para la condición de flujo sobre-expandido. Sin embargo, para otras condiciones del flujo, para un mismo tipo de tobera, el conocimiento es limitado. En el presente trabajo, el objetivo es determinar el comportamiento del flujo en la longitud de garganta y en la divergente, para una tobera cónica experimental clasificada como Helios-X, para la condición de flujo sub-expandido. Se realizaron simulaciones numéricas $2 \mathrm{D}$ del campo de flujo con el código ANSYS-Fluent versión 12.1, aplicando el modelo RANS. Se emplearon las ecuaciones gobernantes para el flujo compresible, conservación de la masa, cantidad de movimiento, energía y de estado; así como, para la turbulencia el modelo SST $k-\omega$ de Menter y para la viscosidad en función de la temperatura la ecuación de Sutherland.

\begin{abstract}
Numerical studies of the flow field for convergentdivergent nozzles with throat length, have reported fluctuations of the flow with oblique shock waves in the throat section, for the over-expanded flow condition. However, for other flow conditions, for the same type of nozzle, knowledge is limited. In the present work, the objective is to determine the behavior of the flow in the throat length and in the divergent, for an experimental conical nozzle classified as Helios-X, for the under-expanded flow condition. 2D numerical simulations of the flow field were performed with the ANSYS-Fluent version 12.1 code, applying the RANS model. The governing equations for compressible flow, conservation of mass, momentum, energy, and state were used; as well as, for turbulence, the Menter model SST $k-\omega$ and for the viscosity as a function of temperature the Sutherland equation.
\end{abstract}

\footnotetext{
${ }^{1}$ Universidad Nacional Experimental Politécnica Antonio José de Sucre, Vice-Rectorado Puerto Ordaz, Venezuela.

${ }^{2, *}$ Grupo de Modelamiento Matemático y Simulación Numérica, Universidad Nacional de Ingeniería, Lima, Perú. Autor para correspondencia : sanluist@gmail.com. (D) http://orcid.org/0000-0001-6320-6864,

${ }^{3}$ Cohetería experimental amateur, Canadá. (D) http://orcid.org/0000-0002-7759-7162

${ }^{4}$ Departamento de Ingeniería Mecánica, Universidad Nacional Experimental Politécnica Antonio José de Sucre Vice-Rectorado Puerto Ordaz, Bolívar, Venezuela. (D) http://orcid.org/0000-0002-0170-2448

${ }^{5}$ Facultad de Ingeniería de Petróleo Gas y Petroquímica, Universidad Nacional de Ingeniería, Lima, Perú.

(D) http://orcid.org/0000-0002-5614-5853
}

Forma sugerida de citación: Tolentino Masgo, S. L. B.; Nakka, R.; Caraballo, S. y Mírez, J. (2021). «Simulación numérica del flujo subexpandido en la tobera cónica experimental helios-x». INGENIUS. N. ${ }^{\circ} 25$, (enero-junio). pp. 81-93. DOI: https://doi.org/10.17163/ings.n25.2021.08. 
En la sección de la garganta, adyacente a la pared, el flujo presentó fluctuaciones, en la simetría axial el flujo presentó una aceleración escalonada; en la sección divergente, el flujo se desaceleró en cierta región, sin embargo, el flujo salió de la tobera a velocidad supersónica ligeramente mayor de Mach 3. Se concluye que en la sección de la longitud de garganta se presenta un patrón de flujo, así como, en la sección divergente.

Palabras clave: garganta, fluctuación, subexpandido, simulación, tobera
In the section of the throat, adjacent to the wall, the flow presented fluctuations, in the axial symmetry the flow presented a stepped acceleration; in the divergent section, the flow slowed in a certain region, however, the flow exited the nozzle at a supersonic speed slightly greater than Mach 3 . It is concluded that in the throat length section there is a flow pattern, as well as, in the divergent section.

Keywords: Throat, Fluctuation, Under-expanded, Simulation, Nozzle. 


\section{Introducción}

El flujo en toberas de motores de cohetes supersónicos es estudiado de manera recurrente para diferentes configuraciones geométricas del perfil interno de las paredes de la tobera. La sección divergente de la tobera puede ser de geometría cónica, de campana, parabólica, rectangulares, planas, entre otras. Tomar datos experimentales en un banco de prueba en estática del flujo dentro de la tobera tiene limitaciones, cuando el flujo de gas está muy por arriba de la temperatura del ambiente. Por lo cual, durante el ensayo experimental registran los datos con instrumentos sensibles a los cambios de presión, temperatura, vibraciones, en la cámara de combustión, al inicio de la tobera, en las paredes, y mediante capturas de imágenes de la región del flujo que se descarga al ambiente. Cuando el flujo está estrangulado en la garganta y basándose a la presión de la cámara de combustión, el flujo puede estar sobreexpandido, óptimamenteexpandido o subexpandido [1]. Señalando que, para un flujo sobreexpandido el choque se presenta dentro de la tobera y para un flujo subexpandido el choque se presenta fuera de ella.

Los datos experimentales obtenidos en la frontera de la tobera, son utilizados para reproducir el comportamiento del campo de flujo dentro de la tobera, mediante la aplicación de la dinámica de fluidos computacional (CFD, por sus siglas en inglés) [2,3], el cual permite cuantificar las magnitudes termodinámicas en un dominio computacional del campo de flujo que no es posible obtener por medios experimentales en campo abierto y laboratorios.

La CFD es una herramienta computacional que permite obtener soluciones aproximadas de la realidad del fenómeno físico, si de manera adecuada se toma en cuenta la geometría del dominio computacional, el refinamiento de la malla, el modelo de turbulencia, el establecimiento de los errores durante la etapa de iteración, así como, otros parámetros de control de variables.

Para flujo compresible están sustentados y reportados trabajos sobre las ondas de choque y ondas de expansión de Prandtl-Meyer [4,5], la captura de imagen de las ondas de choque mediante la técnica Schlieren [6], la turbulencia [7], la capa límite [8,9], así como los modelos de turbulencia [10].

Estudios experimentales en estática y en dinámica para toberas convergentes-divergentes con longitud de garganta han sido reportados con fines de investigación, sin embargo, el conocimiento del comportamiento del régimen del flujo que se presenta dentro de dicha toberas con longitud de garganta es prácticamente nulo, ya que escasamente ha sido abordado empleando herramientas computacionales para determinar sus magnitudes termodinámicas.

Un estudio computacional de la simulación del flujo sobreexpandido en una tobera cónica con longitud de garganta y ángulo medio de $11^{\circ}$ en la sección divergente, perteneciente a un motor de cohete sonda para combustible sólido, clasificado como ULA-1A XP [11], reportó resultados numéricos de las fluctuaciones de la velocidad del flujo y de los choques oblicuos que se presentaron en la sección de la garganta, en el rango de velocidad transónica, así como el comportamiento de la fluctuación del flujo desde el centro hacia las paredes de la garganta. Acotando que, previamente, este tipo de tobera fue ensayada en estática y en dinámica por el Grupo de Ciencias de la Atmósfera y del Espacio (GCAE), de la Universidad de Los Andes, Venezuela [12-14].

En otro estudio realizado, para un flujo subexpandido en una tobera cónica con longitud de garganta y ángulo medio de $15^{\circ}$ en la sección divergente, identificada como tobera Helios-X [15], que también fue simulado el campo de flujo, expusieron sus resultados según el número de Mach, donde, el flujo en la sección de la garganta presentó desaceleración. Aunque aportan un dato fundamental de la velocidad del flujo de acuerdo con el número de Mach, aún queda por analizar con mayor detalle, el campo de flujo para otros parámetros termodinámicos. Por lo cual, es de interés que se debe continuar con la investigación para este tipo de tobera, para determinar el comportamiento del campo de densidad, presión, temperatura, velocidad, asimismo el número de Mach, en todo el dominio, y enfocando con mayor interés en la sección de la garganta, que podrían aportar y enriquecer el conocimiento de la distribución de los gradientes de los parámetros termodinámicos.

La tobera Helios-X, diseñada por Nakka [16], fue ensayada en un banco de prueba en estática y en dinámica. En la Figura 1 se muestra el registro del ensayo en estática de la tobera acoplada al motor cohete Helios-X, el cohete en la plataforma de lanzamiento y el despegue del cohete. Múltiples experimentos realizados por Nakka de toberas en motores de cohetes para combustible sólido de categoría aficionado, en estática y en dinámica, pueden ser obtenidos en su sitio web [16].

En el presente trabajo se ha propuesto continuar con la investigación para el flujo subexpandido en la tobera Helios-X, reportado en [15], con el fin de determinar el comportamiento del flujo en la sección de la garganta y en la divergente. Para lograr el objetivo propuesto, se simuló el campo de flujo supersónico y se obtuvo el campo de presión, número de Mach, velocidad, temperatura y densidad. Los resultados muestran, en la pared, a la entrada de la garganta, una región del flujo que presenta fluctuación, por lo cual, en esa región el flujo acelera y desacelera, en otras regiones, de la misma sección, la velocidad del flujo solo se lentifica.

En la sección 2 se presentan las ecuaciones empleadas, el dominio computacional 2D y el método de solución computacional. En la sección 3 se exponen los 
resultados obtenidos y las discusiones. En la sección 4 se exponen las conclusiones del análisis realizado.

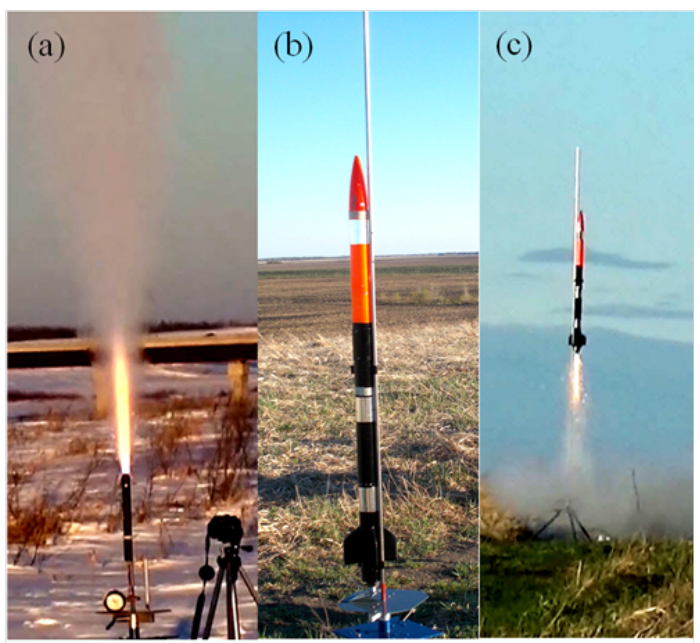

Figura 1. (a) Ensayo experimental en estática de la tobera con longitud de garganta acoplada al motor cohete Helios-X, marzo 2017. (b) Plataforma de lanzamiento del cohete Z-30, con el motor cohete Helios-X instalado. (c) Despegue del cohete Z-30, mayo 2017 [16]

\section{Materiales y métodos}

\subsection{Fundamento matemático}

En el presente trabajo, para la simulación del campo de flujo compresible, se emplea el modelo de las ecuaciones de Navier-Stokes de número de Reynolds promedio (RANS, por sus siglas en inglés). Las ecuaciones gobernantes empleadas son: la ecuación de la conservación de la masa (1), cantidad de movimiento (2), conservación de la energía (3) y de estado (4). En forma compacta y para un flujo en condiciones estacionarias, se expresan como:

$$
\nabla \cdot\left(\rho u_{i}\right)=0
$$

Donde, la densidad es $\rho$ y la velocidad $u$.

$$
\nabla \cdot\left(\rho u_{i} u_{j}\right)=-\nabla p+\nabla(\overline{\bar{\tau}})+\nabla \cdot\left(-\rho \overline{u_{i} u_{j}}\right)
$$

Donde, la presión es $p$; el tensor de tensiones $\overline{\bar{\tau}}$ y las tensiones de Reynolds $-\rho \overline{u_{i} u_{j}}$. Cabe señalar, la ecuación (2) está cerrada, ya que incluye el término de tensiones de Reynolds.

$$
\nabla \cdot\left(u_{i}(\rho E+p)\right)=\nabla \cdot\left(k_{e f f} \nabla T+\left(\overline{\bar{\tau}}_{e f f} \cdot u_{i}\right)\right)
$$

Donde, la energía total es $E$, la temperatura $T$, la conductividad térmica efectiva $k_{e f f}$, y el tensor de tensiones efectivo $\overline{\bar{\tau}}_{e f f}$.

$$
p=\rho R T
$$

Donde, la constante del gas es $R$.

Para flujo compresible, se toman en cuenta las relaciones de presiones (5) y de temperaturas (6) en función del número de Mach el cual es el parámetro dominante, y se expresan como:

$$
\begin{gathered}
\frac{p_{0}}{p}=\left(1+\frac{\gamma-1}{2} M^{2}\right)^{\frac{\gamma}{\gamma-1}} \\
\frac{T_{0}}{T}=1+\frac{\gamma-1}{2} M^{2}
\end{gathered}
$$

Donde, la presión total es $p_{0}$, la temperatura total $T_{0}$, la relación de calores específicos $\gamma$ y el número de Mach, $M$.

El número de Mach supersónico en la salida de la tobera para un flujo subexpansionado y sin choque en la salida de la divergente se determina con la ecuación (7); donde $A / A^{*}$ es la relación de áreas de diseño, el área en la salida de la tobera es $A$ y de la garganta $A^{*}$ :

$$
\frac{A}{A^{*}}=\frac{1}{M}\left(\frac{1+\frac{\gamma-1}{2} M^{2}}{\frac{\gamma+1}{2}}\right)^{\frac{\gamma+1}{2 \gamma-2}}
$$

Las consideraciones del número de Mach son las siguientes: para flujo incompresible $M<0,3$; flujo subsónico $0,3<M<0,8$; flujo transónico $0,8<$ $M<1,2$; flujo supersónico $1,2<M<5$; flujo hipersónico $M>5$; y para el flujo con velocidad sónica, se tiene $M=1$ [5]. Cabe señalar, White [5] considera que a partir de un valor mayor de Mach 3 el flujo es hipersónico, y Anderson [4] a partir de Mach 5.

Para la viscosidad en función de la temperatura (8), de acuerdo con la ley de Sutherland [8], se expresa como:

$$
\frac{\mu}{\mu_{0}}=\left(\frac{T}{T_{0}}\right)^{\frac{3}{2}} \frac{T_{0}+S}{T+S}
$$

Donde, la viscosidad de referencia es $\mu_{0}=$ $1,716 \mathrm{~kg} /(\mathrm{m} \cdot \mathrm{s})$, la temperatura de referencia $T_{0}=$ $273,11 \mathrm{~K}$ y la temperatura efectiva $S=110,56 \mathrm{~K}$.

Para la turbulencia del flujo, se toma en cuenta el modelo de turbulencia SST $k-\omega$ de Menter [17], la cual es resuelta en conjunto con la ecuación de cantidad de movimiento. Este modelo de turbulencia contiene dos ecuaciones, una para la energía cinética específica $k$, y la otra para la tasa de disipación específica $\omega$, por lo cual, logra mejorar las respuestas en presencia de gradientes adversos de presión, y separación de flujo.

El modelo de turbulencia de Menter [17] ha sido comparado con otros modelos de turbulencia para diferentes condiciones del flujo compresible con presencia 
de ondas de choque en diferentes equipos experimentales para flujo sobreexpandido y flujo subexpandido, donde los resultados numéricos para dominios $2 \mathrm{D}$ de geometrías simétricas se superponen sobre los datos experimentales de presión en las paredes de equipos experimentales, en una tobera $[18,19]$ y en un difusor transónico [20,21], siendo las formas de las ondas de choque de los resultados numéricos aproximadamente similares con las experimentales. Aplicaciones del modelo de turbulencia de Menter sustentan su validez para diferentes condiciones del régimen del flujo [22-25]. En dominios 3D, el aporte del análisis físico es mucho mayor, incluso para geometrías asimétricas, y cargas laterales asimétricas en el campo de flujo. Por tanto, para las condiciones del flujo subexpandido y sin condiciones de cargas laterales asimétricas, el modelo de turbulencia de Menter es adecuado para la simulación 2D del flujo subexpandido del presente trabajo.

\subsection{Dominio computacional}

El esquema de la geometría de la tobera cónica experimental convergente-divergente con longitud de garganta, Helios-X [16], se muestra en la Figura 2, y cuyas dimensiones principales y sus respectivas unidades en milímetros se muestran en la misma figura. Siendo longitud de garganta $L_{g}=6,01 \mathrm{~mm}$, diámetro de la garganta $D_{g}=5,58 \mathrm{~mm}$, la relación entre la longitud de garganta y el diámetro $r_{L D}=1,07$, y la relación de áreas de diseño $A / A^{*}=5,206$.

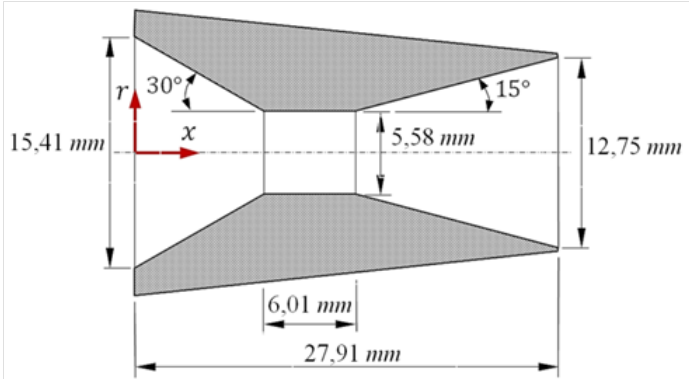

Figura 2. Geometría de la tobera cónica experimental Helios-X [16]

El dominio computacional 2D con simetría axial compuesto por una sección de la cámara de combustión, la tobera y la atmósfera, se muestra en la Figura 3. El propósito de tomar en cuenta una sección corta de la cámara de combustión de 7,47 $\mathrm{mm}$ y no toda la sección del tubo motor, es para aplicar la carga de presión y direccionar el flujo hacia la entrada de la tobera. La longitud de la sección de la tobera cónica es 27,91 $\mathrm{mm}$ y la longitud de la sección del ambiente de la atmósfera $281 \mathrm{~mm}$, respectivamente. Señalando que, la garganta inicia en la posición $x=8,51 \mathrm{~mm}$ y termina en la posición $x=14,53 \mathrm{~mm}$, y su longitud es $L_{g}=6,01 \mathrm{~mm}$.

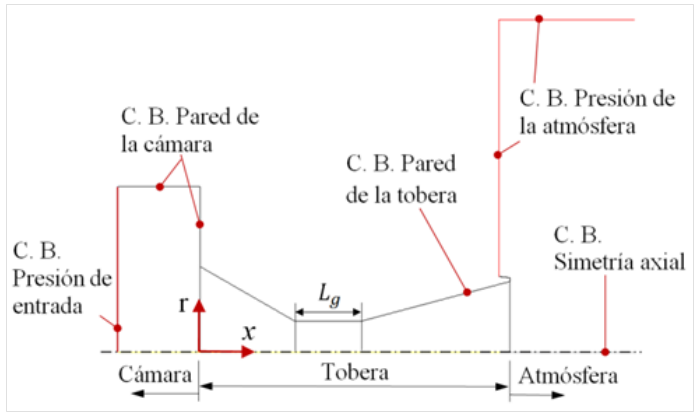

Figura 3. Dominio computacional 2D con simetría axial. El dominio incluye una sección de la cámara de combustión, la tobera y una sección de atmósfera

Además, como la tobera tiene una geometría simétrica de revolución, un dominio 2D es adecuado, lo cual contribuye en la reducción de la cantidad de celdas de la malla y el tiempo de iteración durante el procesamiento de datos computacionales. Además, en la misma figura del dominio se señalan las referencias donde se aplican las condiciones de borde (C.B.).

Las condiciones iniciales y de borde se establecieron como:

En la cámara del motor cohete Helios-X, la presión total absoluta se establece 6996,11 kPa; y de la temperatura total $2558 \mathrm{~K}$.

En el ambiente de la atmósfera, la presión se establece $101,5 k P a$, de la temperatura $263,15 \mathrm{~K}$.

En el eje de simetría axial, la velocidad del flujo en la dirección radial es nula. En las paredes la velocidad es nula por la condición de no deslizamiento.

Las paredes de la sección de la cámara de combustión y de la tobera son consideradas adiabáticas.

El efecto de la gravedad del flujo dentro de la tobera no se considera, esto es debido a la alta velocidad del chorro supersónico en la divergente. En la atmósfera, la velocidad del flujo es supersónico, por lo cual, para la longitud del dominio considerado, el efecto de la gravedad es ínfimo y es considerado despreciable.

Cabe señalar, los datos de presión y de temperatura en la cámara del motor cohete que se aplican en las condiciones de borde del presente trabajo han sido obtenidos por medios experimentales por Nakka [16], así como, los datos del ambiente de la atmósfera registrados con instrumentos de medición de presión y temperatura. Los datos experimentales de la cámara de combustión y del ambiente de la atmósfera aplicados al dominio 2D, contribuyen para obtener la simulación del campo de flujo en todo el dominio, y permiten 
determinar el comportamiento de la presión y temperatura en la pared de la tobera que no ha sido posible medir experimentalmente.

Para el presente trabajo, en las simulaciones numéricas para el presente trabajo, los gases quemados producto de la combustión del propelente sólido compuesto por nitrato de amonio, aluminio, azufre y cloropreno (A24 ANCP) [16], se considera como gas ideal, y como sustancia aire. Como parámetros, se fija la relación de calor específico $\gamma=1,4$, la constante del gas $R=287 \mathrm{~J} /(\mathrm{kg} \cdot K)$, el calor específico a presión constante $C_{p}=1006,43 \mathrm{~J} /(\mathrm{kg} \cdot \mathrm{K})$ y la conductividad térmica $k=0,0242 \mathrm{~W} /(m \cdot K)$.

El dominio computacional mallado se muestra en la Figura 4 y una ampliación de la sección mallada de la tobera se muestra en la misma figura. El mallado se realizó en la plataforma ANSYS-Meshing y se discretizó el dominio mediante la interacción de ICEM-CFD. Se refinó la malla del dominio a lo largo de todas las paredes, por la presencia del esfuerzo cortante en esas regiones. En la sección de la cámara y de la tobera se aplicó un mallado con celdas triangulares, y en la sección del ambiente de la atmósfera un mallado estructurado con celdas cuadriláteras, para un total de 32675 de celdas combinadas. El mallado del dominio mostrado en la figura corresponde para un mallado final, luego de haber realizado un estudio de convergencia numérica para un refinamiento del dominio tres veces.
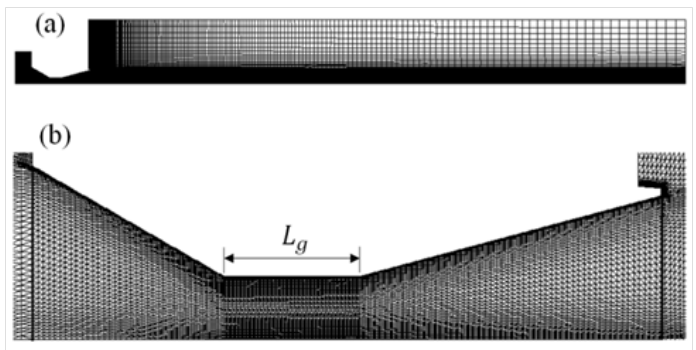

Figura 4. (a) Dominio computacional mallado con un total de 32675 celdas combinadas. (b) Sección del dominio mallado de la tobera.

En el estudio de convergencia numérica, el primer dominio mallado arrojó 30950 celdas, siendo la celda de menor dimensión de $4,9 \times 10^{-5} \mathrm{~mm}$ y de mayor dimensión de $3,37 \times 10^{-4} \mathrm{~mm}$ en la sección de la tobera, y la mayor dimensión de la celda de $3,2 \mathrm{~mm}$ se presentó en la esquina superior derecha en el dominio de la atmósfera. El segundo arrojó 32 296, siendo la celda de menor tamaño de $4,7 \times 10^{-5} \mathrm{~mm}$ y la máxima de $3,31 \times 10^{-4} \mathrm{~mm}$, y en el dominio de la atmósfera la celda máxima de $3,2 \mathrm{~mm}$. El tercero arrojó 32 675 celdas, y con dimensiones de celdas mínima de $4,67 \times 10^{-5} \mathrm{~mm}$ y máxima de $3,29 \times 10^{-4} \mathrm{~mm}$, y en la atmósfera la celda de mayor dimensión de $3,2 \mathrm{~mm}$. Para el primer dominio, el espaciamiento mínimo de la celda en la pared de la tobera, en promedio se obtuvo $y^{+}=0,98$, para el segundo dominio $y^{+}=0,95$ y para el tercer dominio del mallado final $y^{+}=0,94$.

Para los tres casos, la densidad de la malla es alta en la tobera y en la región de la atmósfera donde se presenta el chorro supersónico conocido como pluma. Se tomó como punto de control al final de la sección divergente, en la simetría axial, para evaluar numéricamente el número de Mach, por ser una región crítica por la alta velocidad del flujo, y se obtuvo un error porcentual de 0,04\% entre el tercer dominio mallado y el segundo; y ligeramente mayor, de 0,052 \% entre el segundo y el primero; siendo en la salida de la tobera en la simetría axial del eje $\mathrm{X}$ para el tercer dominio de mallado final y el valor numérico de Mach 3,1.

Para la teoría de flujo cuasiunidimensional con $\gamma=1,4$, y $A / A^{*}=5,206$ de diseño de la tobera Helios-X, y sin choque a la salida de la tobera, se tiene el valor de Mach 3,217 obtenido con la Ecuación (7) (teórica). Siendo la diferencia de magnitud de número de Mach 0,117 entre el cálculo teórico de Mach 3,217 y el cálculo numérico de Mach 3,1 del tercer dominio mallado. Por lo cual, es aceptable la magnitud del resultado de las comparaciones de números de Mach como validación numérica. Para un flujo cuasiunidimensional y subexpandido, el flujo sale de la tobera de manera uniforme y perpendicular al área de salida, por lo cual tiene la misma magnitud en su área de sección transversal, mientras que, para el método numérico el flujo tiene un gradiente de velocidad del número de Mach a la salida de la tobera y su magnitud varía en su área de sección transversal.

El dominio mallado que se presenta en la Figura 4 es adecuado y cumple con los criterios satisfactorios del análisis de convergencia realizado. Aunque los tres dominios satisfacen para $y^{+}<1$, se optó por el dominio mallado con 32675 celdas por tener un mayor refinamiento en las paredes, la cual es empleado en las simulaciones computacionales.

La calidad de la malla, para celdas bidimensionales, y el sesgo equiángulo $\left(Q_{E A S}\right)$, establecen que debe estar $0 \geq Q_{E A S} \geq 1$, para cualquier celda 2D [26]. Para todo el dominio de la malla final, se obtuvo $Q_{E A S}=0,55$. Acotando que, el estudio de convergencia numérica se realizó con el modelo de turbulencia SST $k-\omega$ de Menter [17] para simular la turbulencia del flujo.

\subsection{Método de solución computacional}

Para la simulación del flujo, en el código ANSYSFluent 12.1, el cual aplica el método de volumen finito (MVF), se optó la opción de análisis basado en densidad para un fluido compresible, dominio $2 \mathrm{D}$ con simetría axial en el eje x. Para la turbulencia del flujo se empleó el modelo de SST $k-\omega$ de Menter [17] y para la viscosidad la ecuación de Sutherland [8]. 
En el método de solución, se consideró, la formulación implícita y tipo de flujo Roe-FDS. Para la discretización espacial, el gradiente: Least Squares Cell based; para el flujo, energía cinética turbulenta y la tasa de disipación específica, la opción: First Order Upwin.

En el monitor residual, para el criterio de convergencia absoluta, se estableció un valor fijo de 0,00001, tanto para continuidad, velocidad, y energía. Se realizaron 136400 iteraciones en un tiempo cercano a cincuenta horas, para obtener la convergencia numérica de los resultados finales del campo de flujo, de número de Mach, presión, velocidad, temperatura y densidad.

Para el procesamiento de datos se empleó un equipo con las siguientes características: Laptop marca Síragon, modelo M54R, Intel Core 2 Duo, dos procesadores de 1,8 GHz y memoria RAM de 3 GB.

\section{Resultados y discusión}

En esta sección se presentan los resultados del campo de flujo, para la carga aplicada de presión de entrada de $6996,11 \mathrm{kPa}$ y temperatura de $2558 \mathrm{~K}$, presión del ambiente en la salida de la tobera $101,5 k P a$ y temperatura de $263,15 \mathrm{~K}$.

Las variaciones de las magnitudes de la presión estática (Figura 5), de número de Mach (Figura 6), de velocidad (Figura 7), de temperatura estática (Figura 8) y de la densidad (Figura 9), muestran en qué regiones del dominio alcanzan valores máximos y mínimos. Mediante las líneas de contorno se observa de qué manera se distribuyen en diferentes regiones del campo de flujo, en la sección de la tobera así como en la sección de la atmósfera.

El flujo que sale de la tobera está subexpandido, y se muestra en la atmósfera cómo están conformadas las ondas de choque; y esa región del chorro supersónico donde se presentan las ondas, en la literatura es conocida como pluma.

En la Figura 10 se muestra los perfiles evaluados en la simetría axial, para la región del flujo supersónico que sale de la tobera y descarga en la atmósfera. Se muestran las fluctuaciones producto de la onda de choque, incluso en ciertas regiones la presión cae por debajo de la presión de la atmósfera, acelerando el flujo en esa región antes que se presente el choque a un valor cercano Mach 4,75, y velocidad de $2050 \mathrm{~m} / \mathrm{s}$; mientras que la temperatura cae por debajo de $500 \mathrm{~K}$. Después del choque, el flujo sigue siendo supersónico con presencia de fluctuaciones amortiguadas alrededor de Mach 3, velocidad de $1800 \mathrm{~m} / \mathrm{s}$ y temperatura de $900 \mathrm{~K}$. Además, el comportamiento de las curvas de presión y de densidad muestra una tendencia de equilibrio.

Se han considerado las líneas de contorno, en la sección de la tobera, las cuales aporta mayor infor- mación en cuanto a la distribución de los gradientes de los parámetros termodinámicos. En las figuras ampliadas se ilustra cómo se distribuyen las líneas de contorno en las secciones convergente, de la garganta de longitud $L_{g}$ y en la divergente: para la presión estática (Figura 11), para el número de Mach (Figura 12), para la velocidad (Figura 13), para la temperatura estática (Figura 14) y para la densidad en la (Figura 15). Se observa que las líneas de contorno al inicio de la sección de la garganta tiene un comportamiento distinto a las líneas de contorno al final de la misma garganta, así como, en la parte media.

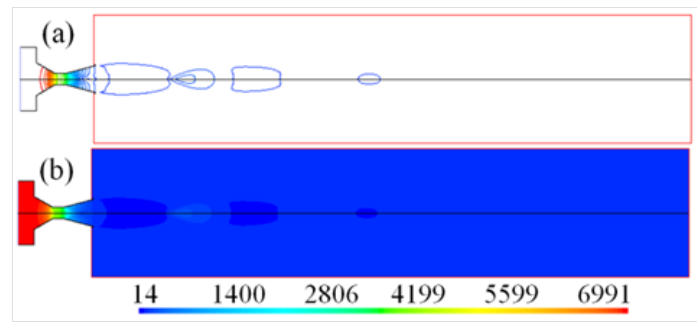

Figura 5. Campo de presión estática $(\mathrm{kPa})$

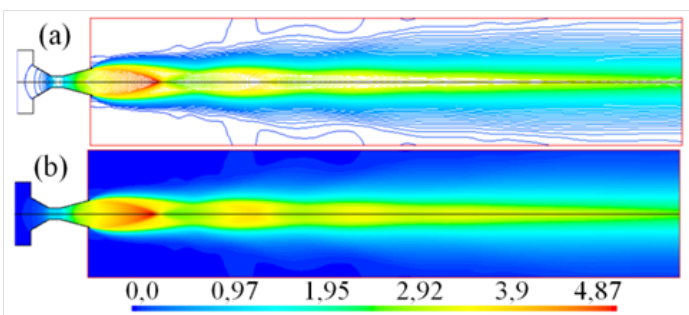

Figura 6. Campo de número de Mach

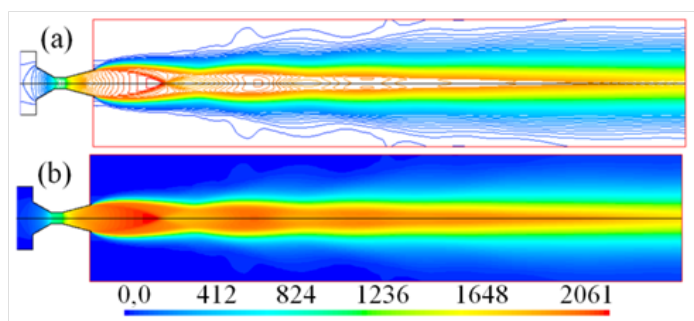

Figura 7. Campo de velocidad (m/s)

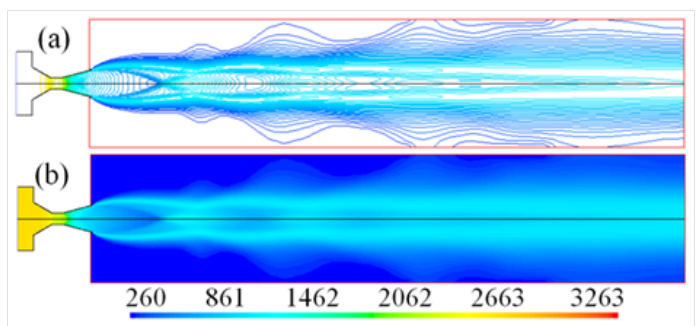

Figura 8. Campo de temperatura estática (K) 


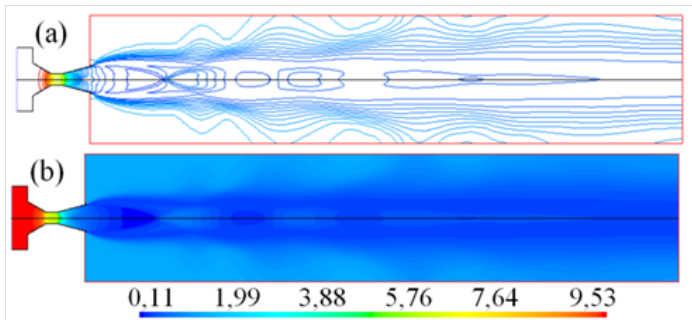

Figura 9. Campo de densidad $\left(\mathrm{kg} / \mathrm{m}^{3}\right)$.

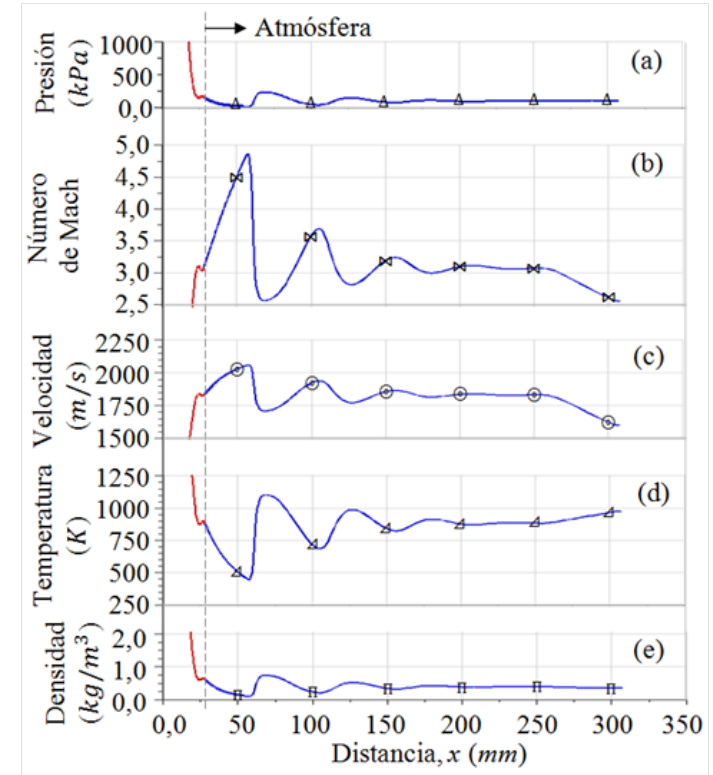

Figura 10. Perfiles evaluados en la simetría axial, en el eje $\mathrm{X}$, en la región del ambiente de la atmósfera. (a) Presión. (b) Número de Mach. (c) Velocidad. (d) Temperatura y (e) Densidad

Cabe señalar, para el caso de la presión estática, se observa que en la parte media de la longitud de garganta, las líneas de contorno tienen una tendencia a ser perpendicular a las paredes de la garganta, por lo cual, la magnitud de la presión en la simetría es similar a la magnitud de la presión en la pared.

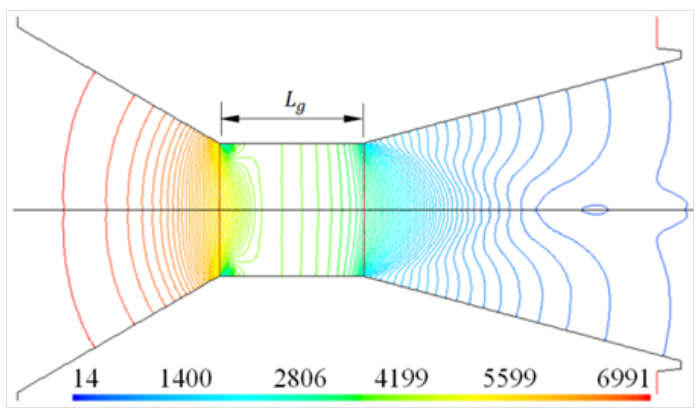

Figura 11. Líneas de contorno de presión estática $(\mathrm{kPa})$ del flujo en la sección de la tobera

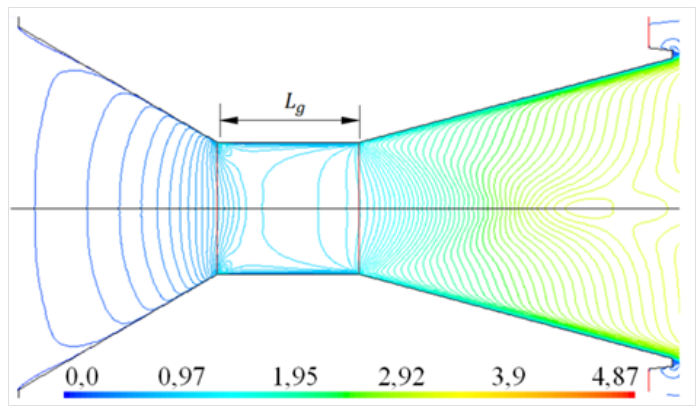

Figura 12. Líneas de contorno de número de Mach del flujo en la sección de la tobera

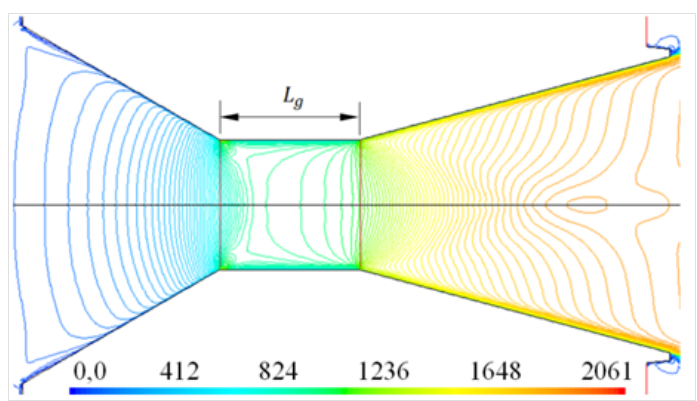

Figura 13. Líneas de contorno de velocidad $(\mathrm{m} / \mathrm{s})$ del flujo en la sección de la tobera

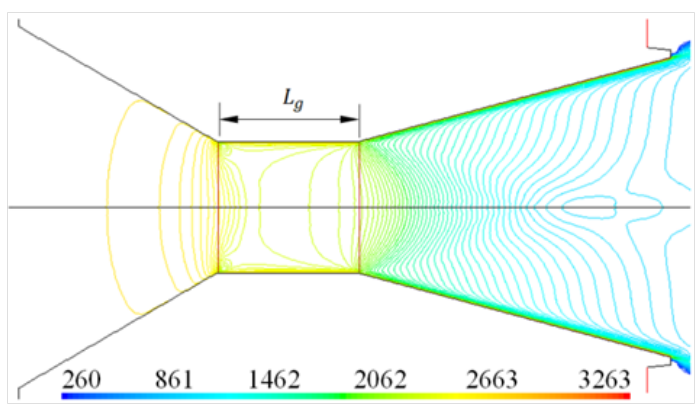

Figura 14. Líneas de contorno de temperatura estática (K) del flujo en la sección de la tobera

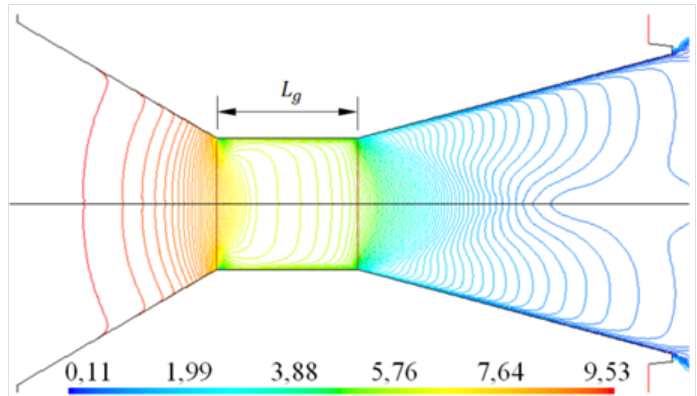

Figura 15. Líneas de contorno de densidad $\left(\mathrm{kg} / \mathrm{m}^{3}\right)$ del flujo en la sección de la tobera

Analizando el comportamiento del flujo desde otra perspectiva, a través de las trayectorias de las curvas numéricas, se muestra que el cambio brusco de la caída 
de presión estática (Figura 16) se presenta en el primer vértice de la sección de la garganta. En la sección media de la garganta, un tramo de las trayectorias de los perfiles de presión en la pared y en la simetría axial es coincidente, lo cual indica que se presenta un régimen de flujo uniforme, con incidencias en la aceleración del flujo. Aguas abajo, a partir de la posición $x=22,5 \mathrm{~mm}$, la caída de presión del flujo tiende a frenar, incrementa ligeramente, después disminuye con menor intensidad, siendo un tramo de la trayectoria del perfil con tendencia oscilatoria; en dicho tramo, las fluctuaciones de presión influyen en el desarrollo de la aceleración del flujo, por lo cual, las magnitudes de los parámetros termodinámicos presentan variaciones.

Para el caso del número de Mach (Figura 17), se muestra la desaceleración de flujo en la sección de la garganta, siendo en la parte media de la sección de la garganta Mach 1, y en la salida de la garganta mayor a este valor. Cerca de la salida de la sección divergente, la velocidad del flujo alcanza un valor mayor de Mach 3, en la posición $x=22,5 \mathrm{~mm}$, aguas abajo se presenta una curvatura, y tiende a disminuir el flujo hasta la salida de la tobera, manteniendo su velocidad mayor a Mach 3, siendo el flujo supersónico. Desde la posición $x=22,5 \mathrm{~mm}$ hasta la salida de la tobera, el comportamiento de la trayectoria de la curva muestra que se produce una desaceleración del flujo antes que salga de la tobera.

En el perfil de velocidad (Figura 18) se observa cómo se comporta el patrón de velocidad en la simetría axial, mas no se presenta en la pared por ser nula la velocidad allí por la condición de no deslizamiento. En la parte media de la sección de la garganta el flujo tiene una velocidad estimada de $920 \mathrm{~m} / \mathrm{s}$; aguas abajo, se muestra cómo se comporta un tramo de la trayectoria del perfil de la velocidad a partir de la posición $x=22,5 \mathrm{~mm}$, con una tendencia de velocidad ligeramente mayor de $1800 \mathrm{~m} / \mathrm{s}$. También como en el caso anterior, para el número de Mach, la velocidad del flujo se desacelera antes de salir de la tobera.

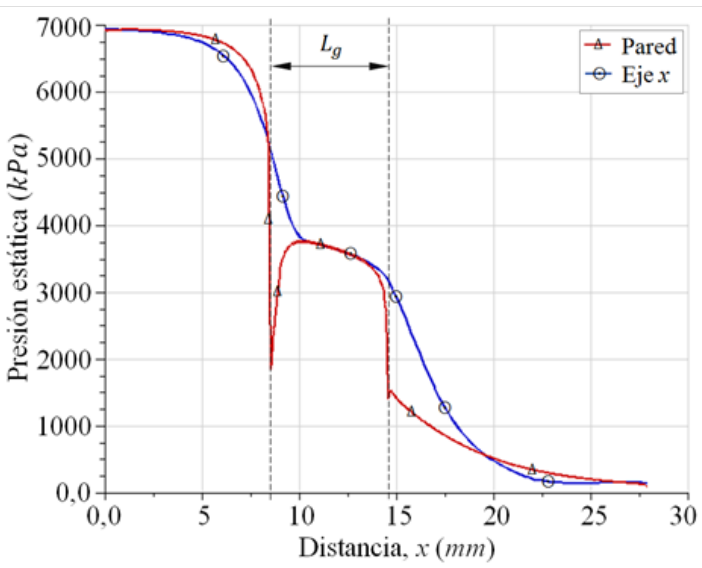

Figura 16. Perfiles de presión estática evaluados en la pared y en el eje X, en la sección de la tobera

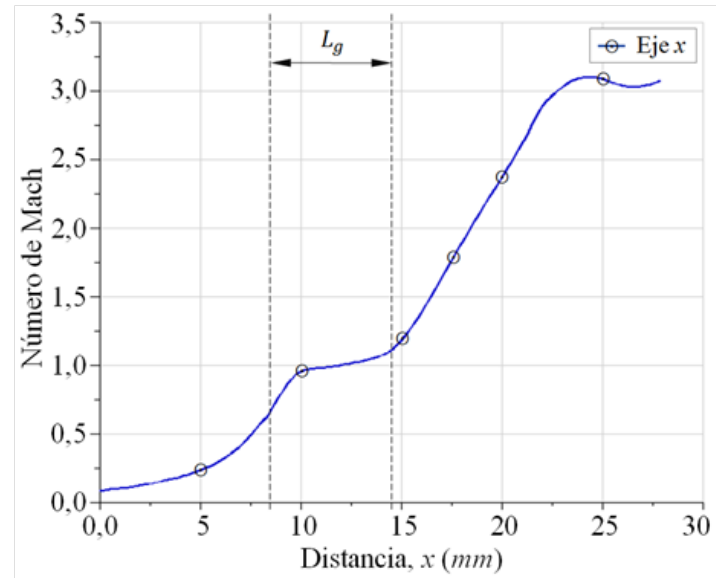

Figura 17. Perfil de número de Mach evaluado en el eje $\mathrm{X}$, en la sección de la tobera

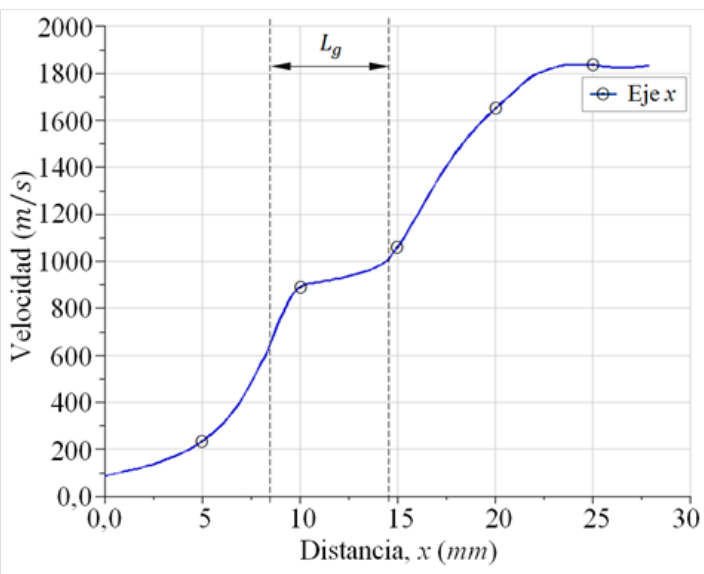

Figura 18. Perfil de velocidad evaluado en el eje X, en la sección de la tobera

Asimismo, para los perfiles de temperatura estática (Figura 19), se muestra el comportamiento de las trayectorias en la pared y en la simetría axial. En la sección de la garganta se observa que la temperatura aumenta y disminuye, e influyen los vértices a sufrir cambios repentinos. En el caso de la sección divergente, el incremento de la temperatura en la pared, a un valor mayor de la temperatura de entrada, es consecuencia de la fricción del flujo a alta velocidad rozando con la pared adiabática, el cual disminuye drásticamente antes de salir el flujo de la tobera. Mientras que, en la simetría axial, la temperatura disminuye producto de la expansión del flujo, y se muestra la fluctuación de su magnitud a partir de la posición $x=22,5 \mathrm{~mm}$. $\mathrm{Y}$ de los perfiles de densidad (Figura 20) muestran su comportamiento en función de la expansión y compresión del flujo, en las secciones convergente, de la garganta y en la divergente.

Los resultados del campo de flujo y de los perfiles que definen la trayectoria de la presión, número de Mach, temperatura, velocidad y densidad, en la 
simetría axial y en la pared de la tobera, son aceptables. Esto se justifica porque la diferencia de magnitud de número de Mach es de 0,117, entre el valor teórico de Mach 3,217 obtenido con la Ecuación (7) y el valor numérico de Mach 3,1 mostrado en la Figura 17, siendo ambos valores calculados a la salida de la tobera y de magnitud cercanos entre sí.

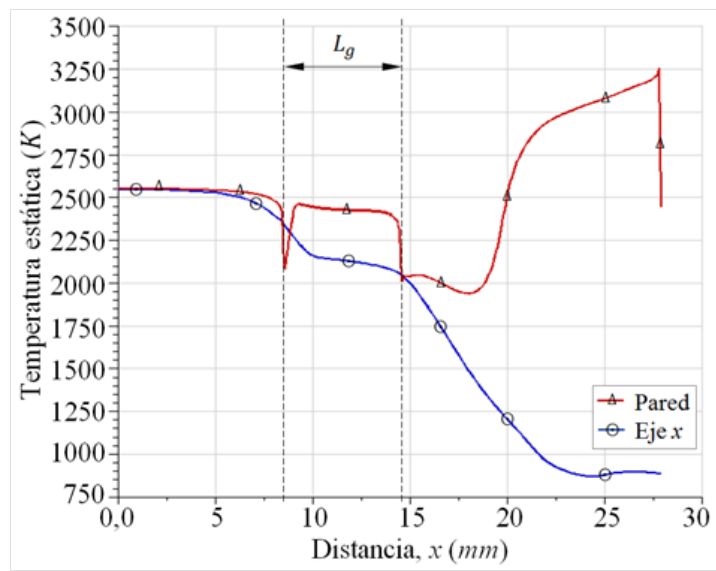

Figura 19. Perfiles de temperatura estática evaluados en la pared y en el eje X, en la sección de la tobera

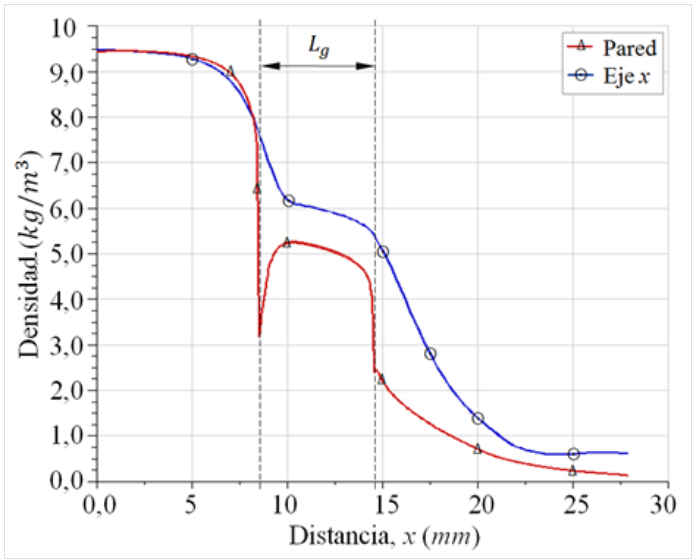

Figura 20. Perfiles de densidad evaluados en la pared y en el eje $\mathrm{X}$, en la sección de la tobera

Los detalles ampliados de la sección de la garganta muestran el comportamiento de las trayectorias de los perfiles de presión estática (Figura 21), número de Mach (Figura 22), velocidad (Figura 23), temperatura estática (Figura 24) y densidad (Figura 25), para diferentes distancias radiales, evaluadas desde la simetría axial hacia la pared. La longitud de garganta inicia en la posición $x=8,51 \mathrm{~mm}$ y termina en la posición $x=14,53 \mathrm{~mm}$. Los perfiles muestran el comportamiento de la región del flujo en el primer vértice, al inicio de la longitud de garganta, a lo largo de la longitud de garganta, así como, cuando el flujo ingresa al inicio de la sección divergente.
Para el caso de número de Mach (Figura 22) y la velocidad (Figura 23), para la región del flujo adyacente a la pared de la sección de la garganta, el flujo se acelera, desacelera y vuelve acelerar; mientras que, en la simetría axial, el flujo se acelera, desacelera y vuelve a acelerar al ingresar a la sección divergente; y esto es consecuencia de las variaciones de presión estática que se presentan desde el inicio de la sección de la garganta hasta el final de dicha sección (ver Figura 21). Por lo cual, la geometría del perfil aerodinámico de la sección de la garganta de longitud $L_{g}$, determina el comportamiento del patrón del flujo en dicha sección, en este caso, se tiene un patrón de flujo en la sección de la garganta, para un flujo subexpansionado en la salida de la tobera a velocidad supersónica mayor de Mach 3.

Las variaciones de la temperatura estática en la sección de la garganta se muestran en la Figura 24, donde en la pared se presenta un incremento de su magnitud, y variaciones en los vértices a la entrada y salida de la garganta (Figura 24). Asimismo, para el caso de la densidad (Figura 25), hacia la pared disminuye su magnitud, y en el vértice a la entrada de la garganta, la densidad disminuye e incrementa, con un comportamiento similar a la trayectoria del perfil de la presión.

Los resultados muestran que la geometría de la sección de la garganta con relación de longitud de garganta y diámetro $r_{L D}=1,07$, inicia en la posición $x=8,51 \mathrm{~mm}$ y termina en la posición $x=14,53 \mathrm{~mm}$, la cual es un tramo corto de sección circular, influye en el desarrollo del flujo en las regiones adyacentes a las paredes, en los vértices y en la simetría. En la simetría axial, en el eje X, el flujo alcanzó un valor en el rango de Mach 0,65 a 1,2, con regiones de velocidad subsónica, transónica y sónica, sin presencia de choque; por lo cual, se presenta un patrón de flujo.

En un trabajo reportado de la tobera cónica experimental ULA-1A XP [11], con ángulo medio de la sección divergente de $11^{\circ}$ y relación longitud de garganta y diámetro $r_{L D}=1,10$, presentó desaceleración del flujo con presencia de ondas de choque oblicuas, en el rango de Mach 0,8 a 1,4; para un flujo sobreexpandido.

Al comparar ambos casos, para el flujo en la sección de la garganta, la longitud de la misma influye en el desarrollo del flujo, sea un flujo subexpandido o sobreexpandido.

Otro patrón de flujo se presentó a partir de la posición $x=22,5 \mathrm{~mm}$. Desde la posición de $x=22,5 \mathrm{~mm}$ hasta la salida de la tobera, el comportamiento de la trayectoria de la curva muestra que se produce una desaceleración del flujo en la divergente, luego que el flujo alcanza la velocidad supersónica Mach 3.

Resultados similares de la desaceleración del flujo en la divergente para dominios $2 \mathrm{D}$, fue reportado en [19] mediante un patrón de densidad, para un ángulo 
medio de $11,01^{\circ}$ de una tobera plana, para condiciones de flujo sobre-expandido. Otro estudio reportó desaceleración del flujo para una tobera cónica de ángulo medio menor de $5^{\circ}$ [27], así como, para una tobera con contorno optimizado y de contorno parabólico [28].

Por lo cual, no es un resultado aislado de la fluctuación obtenido en el presente trabajo, al presentar una región del flujo una desaceleración en la sección divergente, con un patrón definido.

Cabe destacar, en la literatura para flujo unidimensional invíscido $[1,4,5,9,26]$, detallan el incremento de la velocidad del flujo de acuerdo con el número de Mach en la divergente de una tobera, la trayectoria de la curva es creciente y tiende a curvarse hacia la salida de la misma, y no presenta fluctuación alguna. Sin embargo, los resultados del presente trabajo muestran que se produce una fluctuación para cierta región del flujo en la divergente, a pesar de que la onda de choque se presenta fuera de la tobera.

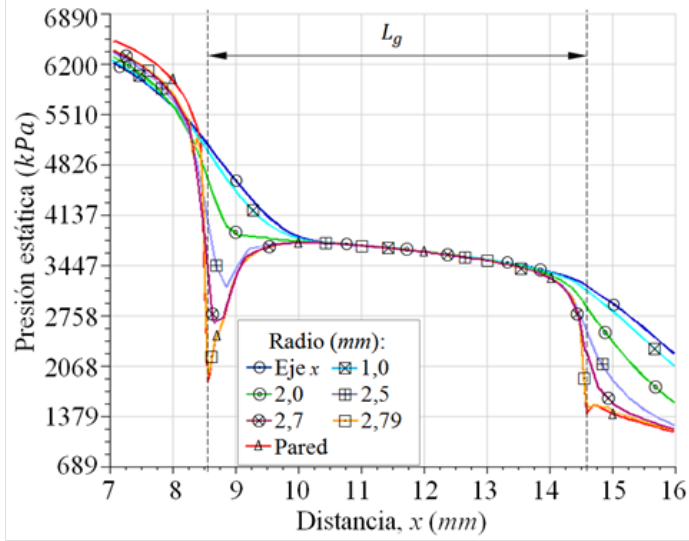

Figura 21. Perfiles de presión estática evaluados en la sección de la garganta de la tobera

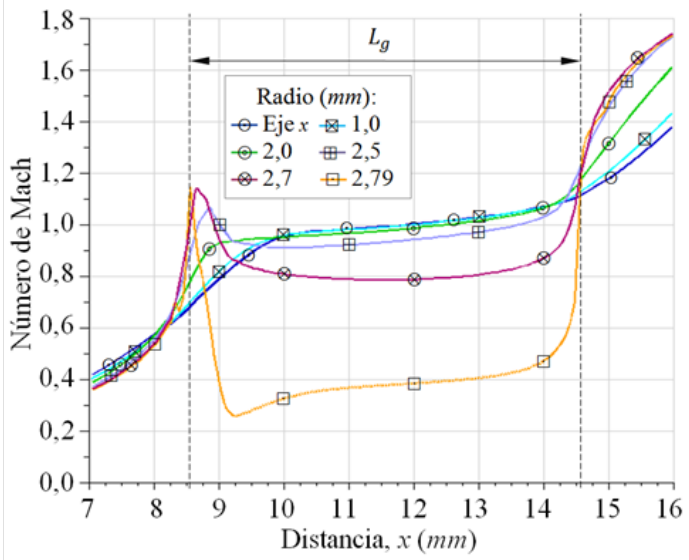

Figura 22. Perfiles de número de Mach evaluados en la sección de la garganta de la tobera

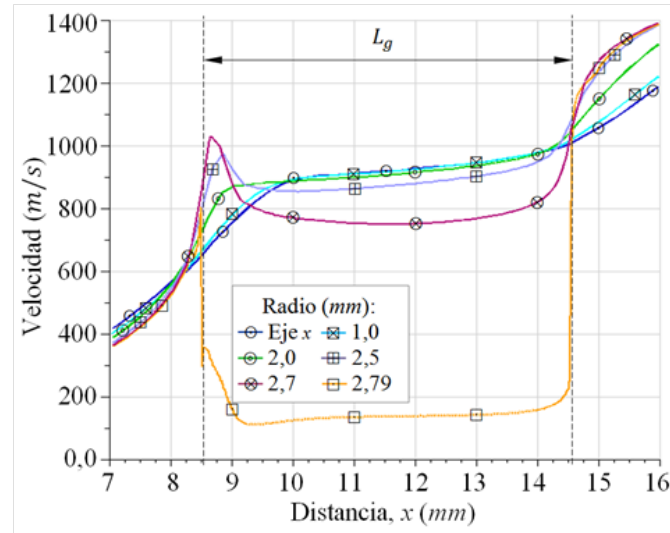

Figura 23. Perfiles de velocidad evaluados en la sección de la garganta de la tobera

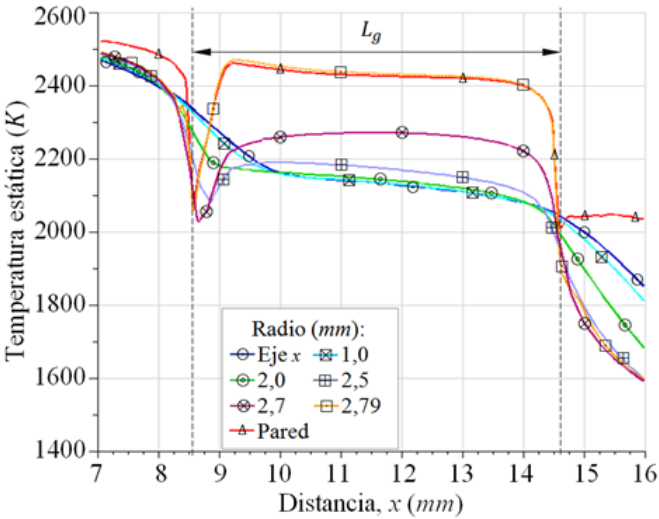

Figura 24. Perfiles de temperatura estática evaluados en la sección de la garganta de la tobera

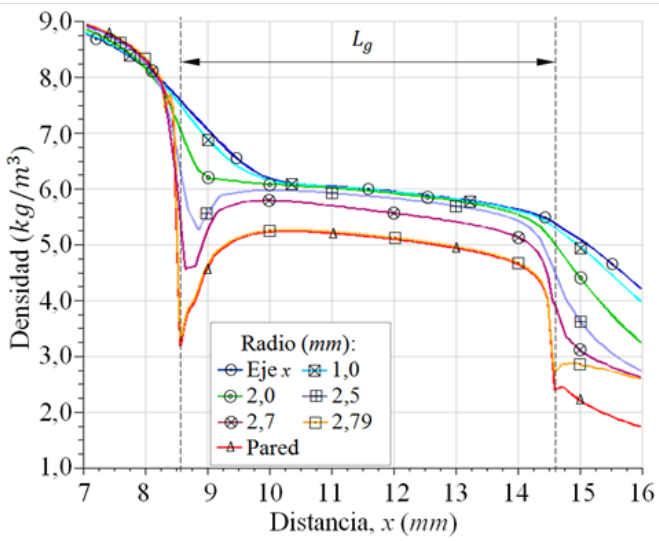

Figura 25. Perfiles de densidad evaluados en la sección de la garganta de la tobera

\section{Conclusiones}

De acuerdo con los análisis realizados, de los resultados de las simulaciones numéricas del flujo subexpansionado, se concluye que: 
En la sección de la tobera, se presentan dos regiones donde la caída de presión del flujo se lentifica, en la sección de longitud de garganta, que se encuentra entre la posición $x=8,51 \mathrm{~mm}$ y $x=14,53 \mathrm{~mm}$; y al final de la sección divergente, a partir de la posición $x=22,5 \mathrm{~mm}$ hasta la salida de la tobera. Por lo cual, en ambas regiones, el flujo se desacelera.

En la simetría axial, al final de la sección divergente de la tobera, el flujo alcanza un valor ligeramente mayor de Mach 3, y velocidad del flujo de $1800 \mathrm{~m} / \mathrm{s}$. En el ambiente de la atmósfera alcanza un valor estimado de Mach 4,75, y velocidad del flujo de $2050 \mathrm{~m} / \mathrm{s}$, antes que se produzca el choque. Aguas abajo, las magnitudes de número de Mach y de velocidad fluctúan, hasta una distancia prolongada, alrededor de Mach 3.

Los perfiles de presión estática, número de Mach, velocidad, temperatura estática y densidad, muestra cómo se desarrolla el flujo en la sección de la garganta, donde al inicio de la sección de la garganta, está presente el vértice; una región del flujo adyacente a la pared presenta fluctuaciones, ocasionando caídas de presión, por lo cual, la velocidad del flujo en esa región adyacente a la pared se acelera y desacelera.

En la simetría axial, en la garganta, en la posición $x=8,51 \mathrm{~mm}$ el flujo alcanza un valor estimado de Mach 0,68, al final de la sección de la garganta, en la posición $x=14,53 \mathrm{~mm}$ un valor estimado de Mach 1,1; por lo cual, una región del flujo es subsónica y el resto transónica. En dicha sección de la garganta, la trayectoria del perfil define un comportamiento escalonado.

Se considera un trabajo en el futuro, para dominios $2 \mathrm{D}$ y $3 \mathrm{D}, \mathrm{y}$ con diferentes códigos computacionales, reducir de manera progresiva la longitud de garganta $L_{g}$, para determinar si persiste la influencia en la aceleración del flujo o si se presenta una posible fluctuación que origine ondas de choque oblicuas. Asimismo, determinar si la fluctuación de los parámetros termodinámicos, tales como el número de Mach, presión y temperatura, en la simetría axial, al final de la sección divergente presenta algún cambio significativo.

\section{Agradecimientos}

Mi agradecimiento a Jehová, mi Dios todopoderoso, mi fuente de sabiduría e inspiración. Al Centro de Estudios Energéticos de la Universidad Nacional Experimental Politécnica «AJS» Vice-Rectorado Puerto Ordaz (UNEXPO), Bolívar, Venezuela. Al Grupo de Modelamiento Matemático y Simulación Numérica (GMMNS, Group of Mathematical Modeling and Numerical Simulation) de la Universidad Nacional de Ingeniería (UNI), Lima, Perú. A Richard Nakka (Ingeniero Aeroespacial), por el aporte de datos experimentales de la tobera del motor cohete Helios-X.

\section{Referencias}

[1] G. P. Sutton and O. Biblarz, Rocket propulsion elements. John Wiley \& Sons, 2016. [Online]. Available: https://bit.ly/35psFaR

[2] J. Blazek, Computational fluid dynamics: principles and applications. Butterworth-Heinemann, 2015. [Online]. Available: https://bit.ly/3pnxjhx

[3] B. Andersson, R. Andersson, L. Hakansson, M. Mortensen, R. Sudiyo, and B. van Wachem, Computational Fluid Dynamics Engineers. Cambridge University Press, 2011. [Online]. Available: https://bit.ly/32BPCWo

[4] J. D. Anderson, Fundamentals of aerodynamics. McGraw-Hill international editions. Mechanical engineering series, 1984. [Online]. Available: https://bit.ly/3eQR5ge

[5] F. M. White, Fluid Mechanics. McGraw-Hill series in mechanical engineering, 2011. [Online]. Available: https://bit.ly/35opmAy

[6] P. Krehl and S. Engemann, "August toepler the first who visualized shock waves," Shock Waves, vol. 5, no. 1, pp. 1-18, Jun. 1995. [Online]. Available: https://doi.org/10.1007/BF02425031

[7] V. Karman, "The fundamentals of the statistical theory of turbulence," Journal of the Aeronautical Sciences, vol. 4, no. 4, pp. 131-138, 1937. [Online]. Available: https://doi.org/10.2514/8.350

[8] F. White, Viscous fluid flow. McGraw-Hill series in Aeronautical and Aerospace Engineering, 1974. [Online]. Available: https://bit.ly/3eRRCyP

[9] H. Schlichting and K. Gersten, Boundary-Layer Theory. Springer, 2016. [Online]. Available: https://bit.ly/36yZGAx

[10] D. C. Wilcox, Turbulence Modeling for CFD. DCW Industries, Incorporated, 1994. [Online]. Available: https://bit.ly/32HZnCm

[11] A. L. Tolentino, J. Ferreira, M. Parco, L. Lacruz, and V. Marcano, "Simulación numérica del flujo sobre-expandido en la tobera cónica experimental ULA-1A XP," Unviversidad, Ciencia y Tecnología, vol. 21, no. 84, pp. 126-133, 2017. [Online]. Available: https://bit.ly/2H4yX6k

[12] V. Marcano, P. Benitez, C. La Rosa, L. La Cruz, M. A. Parco, J. Ferreira, R. Andrenssen, A. Serra Valls, M. Peñaloza, L. Rodríguez, J. E. Cárdenas, V. Minitti, and J. J. Rojas, "Progresos alcanzados en el proyecto universitario cohete sonda ULA," Universidad, Ciencia y Tecnología, vol. 13, no. 53, pp. 305-316, 2009. [Online]. Available: https://bit.ly/3f73vB2 
[13] L. Lacruz-Rincón, M. A. Parco-Brizuela, R. Santos-Luque, C. Torres-Monzón, J. FerreiraRodríguez, and P. Benítez-Díaz, "Análisis experimental de las oscilaciones de presión interna en un motor de combustible solido para cohete sonda," Ciencia e Ingeniería, vol. 13, no. 53, 2016. [Online]. Available: https://bit.ly/3noQfdL

[14] Universidad de los Andes. Programa espacial ULA. [Online]. Available: https://bit.ly/35tiodw

[15] S. L. Tolentino Masgo and R. Nakka, "Simulación del flujo supersónico en la tobera del motor cohete Helios-X de categoría amateur," in Jornadas de Investigación, 2019. [Online]. Available: https://bit.ly/3pwDY8U

[16] R. Nakka. Richard Nakka's experimental rocketry web site. [Online]. Available: https://bit.ly/2IrDZKX

[17] F. R. Menter, "Two equation eddy-viscosity turbulence models for engineering applications," Aerospace Research Central, vol. 32, no. 8, pp. 1598-1605, 2012. [Online]. Available: https://doi.org/10.2514/3.12149

[18] A. Balabel, A. M. Hegab, M. Nasr, and S. M. ElBehery, "Assessment of turbulence modeling for gas flow in two-dimensional convergent-divergent rocket nozzle," Applied Mathematical Modelling, vol. 35, no. 7, pp. 3408-3422, 2011. [Online]. Available: https://doi.org/10.1016/j.apm.2011.01.013

[19] S. L. Tolentino Masgo, "Evaluación de modelos de turbulencia para el flujo de aire en una tobera plana," INGENIUS, no. 22, pp. 25-37, 2019. [Online]. Available: https://doi.org/10.17163/ings.n22.2019.03

[20] Y. Liu, J. Wu, and L. Lu, "Performance of turbulence models for transonic flows in a diffuser," Modern Physics Letters B, vol. 30, no. 25, p. 1650326, 2016. [Online]. Available: https://doi.org/10.1142/S0217984916503267

[21] S. L. B. Tolentino Masgo, "Evaluación de modelos de turbulencia para el flujo de aire en un difusor transónico," Revista Politécnica, vol. 45, no. 1, pp. 25-38, abr. 2020. [Online]. Available: https://doi.org/10.33333/rp.vol45n1.03

[22] Y. Zhang, H. Chen, M. Zhang, M. Zhang, Z. Li, and S. Fu, "Performance prediction of conical nozzle using navier-stokes computation," Journal of Propulsion and Power, vol. 31, no. 1, pp. 192-203, 2015. [Online]. Available: https://doi.org/10.2514/1.B35164

[23] R. Jia, Z. Jiang, and W. Zhang, "Numerical analysis of flow separation and side loads of a conical nozzle during staging," Proceedings of the Institution of Mechanical Engineers, Part G: Journal of Aerospace Engineering, vol. 230, no. 5, pp. 845-855, 2016. [Online]. Available: https://doi.org/10.1177/0954410015599798

[24] H. Ding, C. Wang, and G. Wang, "Transient conjugate heat transfer in critical flow nozzles," International Journal of Heat and Mass Transfer, vol. 104, pp. 930-942, 2017. [Online]. Available: https://doi.org/10.1016/j. ijheatmasstransfer.2016.09.021

[25] A. K. Mubarak and P. S. Tide, "Design of a double parabolic supersonic nozzle and performance evaluation by experimental and numerical methods," Aircraft Engineering and Aerospace Technology, vol. 91, no. 1, pp. 145-156, Dec. 2020. [Online]. Available: https://doi.org/10.1108/AEAT-12-2017-0275

[26] R. H. Pletcher, J. C. Tannehill, and D. Anderson, Computational Fluid Mechanics and Heat Transfer. CRC Press, 2012. [Online]. Available: https://bit.ly/3psYKX9

[27] D. Munday, E. Gutmark, J. Liu, and K. Kailasanath, Flow Structure of Supersonic Jets from Conical C-D Nozzles. [Online]. Available: https://doi.org/10.2514/6.2009-4005

[28] J. Östlund and B. Muhammad-Klingmann, "Supersonic Flow Separation with Application to Rocket Engine Nozzles ," Applied Mechanics Reviews, vol. 58, no. 3, pp. 143-177, 05 2005. [Online]. Available: https://doi.org/10.1115/1.1894402 